\title{
Once-daily single-inhaler versus twice-daily multiple-inhaler triple therapy in patients with COPD: lung function and health status results from two replicate randomized controlled trials
}

Gary T. Ferguson ${ }^{1}$, Nicola Brown ${ }^{2}$, Chris Compton ${ }^{3}$, Thomas C. Corbridge ${ }^{4,5}$, Kelly Dorais ${ }^{6}$, Charles Fogarty ${ }^{7}$, Catherine Harvey ${ }^{2}$, Morrys C. Kaisermann ${ }^{6}$, David A. Lipson ${ }^{6,8}$, Neil Martin ${ }^{3,9}$, Frank Sciurba ${ }^{10}$, Marjorie Stiegler ${ }^{4,11}$, Chang-Qing Zhu ${ }^{2}$ and David Bernstein ${ }^{12^{*}}$

\begin{abstract}
Background: The comparative efficacy of inhaled corticosteroid/long-acting muscarinic antagonist/long-acting $\beta_{2^{-}}$ agonist (ICS/LAMA/LABA) triple therapy administered via single or multiple inhalers in patients with chronic obstructive pulmonary disease (COPD) has not been evaluated comprehensively. We conducted two replicate trials comparing single- with multiple-inhaler ICS/LAMA/LABA combination in COPD.
\end{abstract}

Methods: 207608 and 207609 were Phase IV, 12-week, randomized, double-blind, triple-dummy non-inferiority trials comparing once-daily fluticasone furoate/umeclidinium/vilanterol (FF/UMECNI) 100/62.5/25 $\mu \mathrm{g}$ via Ellipta inhaler, with twice-daily budesonide/formoterol (BUD/FOR) 400/12 $\mu \mathrm{g}$ via metered-dose inhaler plus once-daily tiotropium (TIO) $18 \mu \mathrm{g}$ via HandiHaler. Patients had symptomatic COPD and forced expiratory volume in $1 \mathrm{~s}\left(\mathrm{FEV}_{1}\right)<50 \%$ predicted, or $\mathrm{FEV}_{1}<80 \%$ predicted and $\geq 2$ moderate or 1 severe exacerbations in the prior year. The primary endpoint in both trials was weighted mean change from baseline (wmCFB) in 0-24-h FEV 1 at Week 12. Secondary endpoints included CFB in trough FEV ${ }_{1}$ at Day 84 and 85 . Other endpoints included serial FEV 1 and health status outcomes at Week 12. Safety was evaluated descriptively.

Results: The modified per-protocol population included 720 and 711 patients in studies 207608 and 207609 (intent-to-treat population: 728 and 732). FF/UMECNI was non-inferior to BUD/FOR+TIO for WmCFB in 0-24-h FEV at Week 12 (Study 207608 treatment difference [95\% confidence interval]: $15 \mathrm{~mL}$ [- 13, 43]; Study 207609: $11 \mathrm{~mL}$ $[-20,41])$. FF/UMECNI improved trough FEV 1 CFB versus BUD/FOR+TIO at Day 84 and 85 (Day 85 treatment difference: Study 207608: $38 \mathrm{~mL}[10,66]$; Study 207609: $51 \mathrm{~mL}$ [21, 82]) and FEV ${ }_{1}$ at 12 and $24 \mathrm{~h}$ post-morning dose at Week 12 in both studies. No treatment differences were seen in health status outcomes. Safety profiles were (Continued on next page)

\footnotetext{
* Correspondence: bernstdd@ucmail.uc.edu

${ }^{12}$ Bernstein Clinical Research Center and Division of Immunology, Allergy and Rheumatology, University of Cincinnati College of Medicine, Cincinnati, OH, USA

Full list of author information is available at the end of the article
}

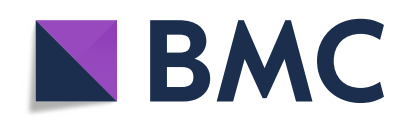

(c) The Author(s). 2020 Open Access This article is licensed under a Creative Commons Attribution 4.0 International License, which permits use, sharing, adaptation, distribution and reproduction in any medium or format, as long as you give appropriate credit to the original author(s) and the source, provide a link to the Creative Commons licence, and indicate if changes were made. The images or other third party material in this article are included in the article's Creative Commons licence, unless indicated otherwise in a credit line to the material. If material is not included in the article's Creative Commons licence and your intended use is not permitted by statutory regulation or exceeds the permitted use, you will need to obtain permission directly from the copyright holder. To view a copy of this licence, visit http://creativecommons.org/licenses/by/4.0/ The Creative Commons Public Domain Dedication waiver (http://creativecommons.org/publicdomain/zero/1.0/) applies to the data made available in this article, unless otherwise stated in a credit line to the data. 


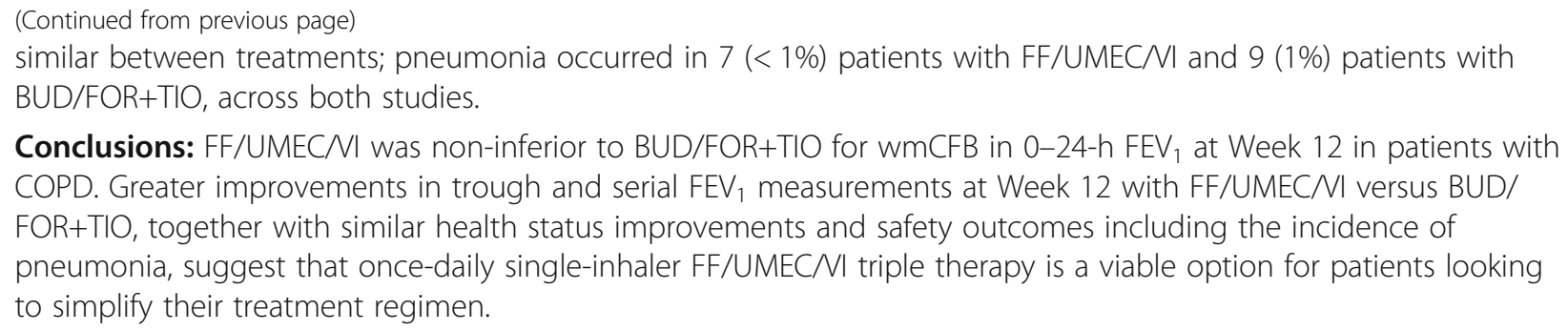

\section{Introduction}

Chronic obstructive pulmonary disease (COPD) is a progressive disease, characterized by persistent respiratory symptoms, including dyspnea, cough, sputum production, and airflow limitation [1]. The 2020 Global Initiative for Chronic Obstructive Lung Disease (GOLD) guidelines recommend treatment initiation based on a patient's symptoms and risk of exacerbations, with treatment escalation determined by the persistence of dyspnea and recurrent exacerbations while on treatment [1].

Escalation to triple therapy with an inhaled corticosteroid (ICS), a long-acting muscarinic antagonist (LAMA), and a long-acting $\beta_{2}$-agonist (LABA) is recommended for patients who continue to experience clinically significant symptoms or recurrent exacerbations while receiving dual LAMA/ LABA or ICS/LABA therapy [1]. Until recently, inhaled triple therapy has required the administration of multiple inhalers, several times a day, with the most commonly administered multiple-inhaler triple therapy in the USA being twice-daily budesonide/formoterol (BUD/FOR) plus oncedaily tiotropium (TIO) [2]. The use of multiple-inhaler triple therapies has demonstrated improvements in lung function, health-related quality of life, hospitalization rates, and rescue medication use in patients with COPD compared with those receiving dual or monotherapies [3-12]. However, outside of the clinical trial environment, adherence to inhaled therapy for COPD is generally low, with non-adherence rates ranging from 50 to $80 \%$ [13-16], and patients with pulmonary diseases demonstrating lower adherence rates than many other chronic conditions [17]. This lack of adherence to medication can have a detrimental effect on patients' well-being, and has been associated with increased risk of hospitalization and mortality, and reduced quality of life and productivity in patients with COPD [18].

Real-world evidence suggests that simplifying inhaler treatment regimens may improve adherence to and persistence with treatment for patients with COPD, potentially resulting in improved health outcomes due to a reduction in treatment discontinuation [18-20]. Although data are limited, some studies have suggested that simplifying treatment with a single inhaler may offer potential advantages in practicality and therapy adherence compared with multiple-inhaler therapy [19-21]. Recently, single inhalers containing ICS/LAMA/LABA have been developed. Phase III studies have demonstrated reductions in exacerbation rates, as well as improvements in health status and lung function, with single-inhaler triple therapy versus dual LAMA/LABA or ICS/LABA therapy, or LAMA monotherapy in patients with symptomatic COPD [12, 22-27]. The TRINITY study (NCT01911364) demonstrated that twice-daily single-inhaler triple therapy with beclometasone dipropionate/glycopyrronium bromide/ formoterol fumarate had similar efficacy and safety as twice-daily beclometasone dipropionate/formoterol fumarate plus TIO multiple-inhaler triple therapy [27]. Nonetheless, further data comparing single-inhaler and multiple-inhaler therapy are needed.

Here, we report the results of two replicate Phase IV trials comparing the efficacy and safety of two ICS/LABA/LAMA combinations, one administered in a single inhaler (once-daily fluticasone furoate/umeclidinium/vilanterol [FF/UMEC/VI]) and one administered via multiple inhalers (twice-daily BUD/FOR plus once-daily TIO).

\section{Methods}

\section{Study design}

Study 207608 (NCT03478683) and Study 207609 (NCT03478696) were two replicate Phase IV, 12-week, randomized, double-blind, triple-dummy, parallel-group, multicenter, non-inferiority trials of single-inhaler triple therapy (FF/UMEC/VI) compared with multiple-inhaler triple combination therapy (BUD/FOR+TIO) in patients with COPD. Both trials were conducted from June 2018 to March 2019, with 59 centers in four countries included in Study 207608 and 58 centers in three countries included in Study 207609.

Following screening (Visit 1) patients entered a 4-week run-in period during which they discontinued all existing 
COPD medications and received BUD/FOR $(400 / 12 \mu \mathrm{g})$ twice daily via metered-dose inhaler (MDI) plus TIO $(18 \mu \mathrm{g})$ once daily via HandiHaler (Boehringer Ingelheim International $\mathrm{GmbH}$ ) plus placebo once daily via Ellipta dry-powder inhaler (GlaxoSmithKline). Following run-in (Visit 2; Day 1 of study), an Interactive Web Response System was used to randomize patients $1: 1$ to receive FF/UMEC/VI 100/62.5/25 $\mu$ g once daily (in the morning) via Ellipta plus two inhalations of placebo twice daily via MDI (in the morning and evening) plus placebo once daily via HandiHaler (in the morning), or two inhalations of BUD/FOR 200/6 $\mu$ g twice daily via MDI (in the morning and evening) plus TIO $18 \mu \mathrm{g}$ once daily via HandiHaler (in the morning) plus placebo once daily (in the morning) via Ellipta for 84 days (Fig. 1). Patients self-administered their randomized treatment at home each day and during two ontreatment clinic visits at Week 4 (Visit 3) and Week 12 (Visit 4). A further safety follow-up telephone call or clinic visit (Visit 5) was conducted approximately 7 days after Visit 4 or end of study, whichever was first. Rescue albuterol was available as needed throughout the study but was withheld for at least $4 \mathrm{~h}$ prior to spirometry assessments.

All patients provided written informed consent. The trials were approved by the relevant ethics committee or institutional review board, in accordance with the International Council for Harmonisation of Technical Requirements for Registration of Pharmaceuticals for Human Use Good Clinical Practice and applicable country-specific requirements.

\section{Study population}

At screening, eligible patients were outpatients $\geq 40$ years of age, who were current or former smokers with a history of $\geq 10$ pack-years with an established clinical history of COPD (as defined by the American Thoracic Society/European Respiratory Society [ATS/ERS] [28]). Patients were also required to have been receiving daily maintenance therapy for $\geq 3$ months, have a postbronchodilator forced expiratory volume in $1 \mathrm{~s}\left(\mathrm{FEV}_{1}\right)$ of $<50 \%$ predicted (or a post-bronchodilator $\mathrm{FEV}_{1}<80 \%$ predicted and $\geq 2$ moderate exacerbations or 1 severe exacerbation in the prior 12 months), a post-bronchodilator $\mathrm{FEV}_{1} /$ forced vital capacity $(\mathrm{FVC})$ ratio $<0.70$ and a COPD Assessment Test (CAT) score $\geq 10$.

Patients with a current diagnosis of asthma or other clinically significant respiratory disorders were excluded, as were those with pneumonia and/or a moderate or severe COPD exacerbation that had not resolved $\geq 14$ days prior to screening and $\geq 30$ days following the last dose of oral/systemic corticosteroid (if applicable), or a respiratory tract infection that had not resolved $\geq 7$ days prior to screening. Patients at risk of non-compliance with study medication or attendance for scheduled visits, or unable to comply with the study procedures were also excluded. Compliance was continually assessed during the trial. Compliance between visits was assessed through querying the participant and recording the number of doses remaining in the Ellipta and MDI or the number of TIO or matching placebo capsules dispensed and taken by each participant and recorded in

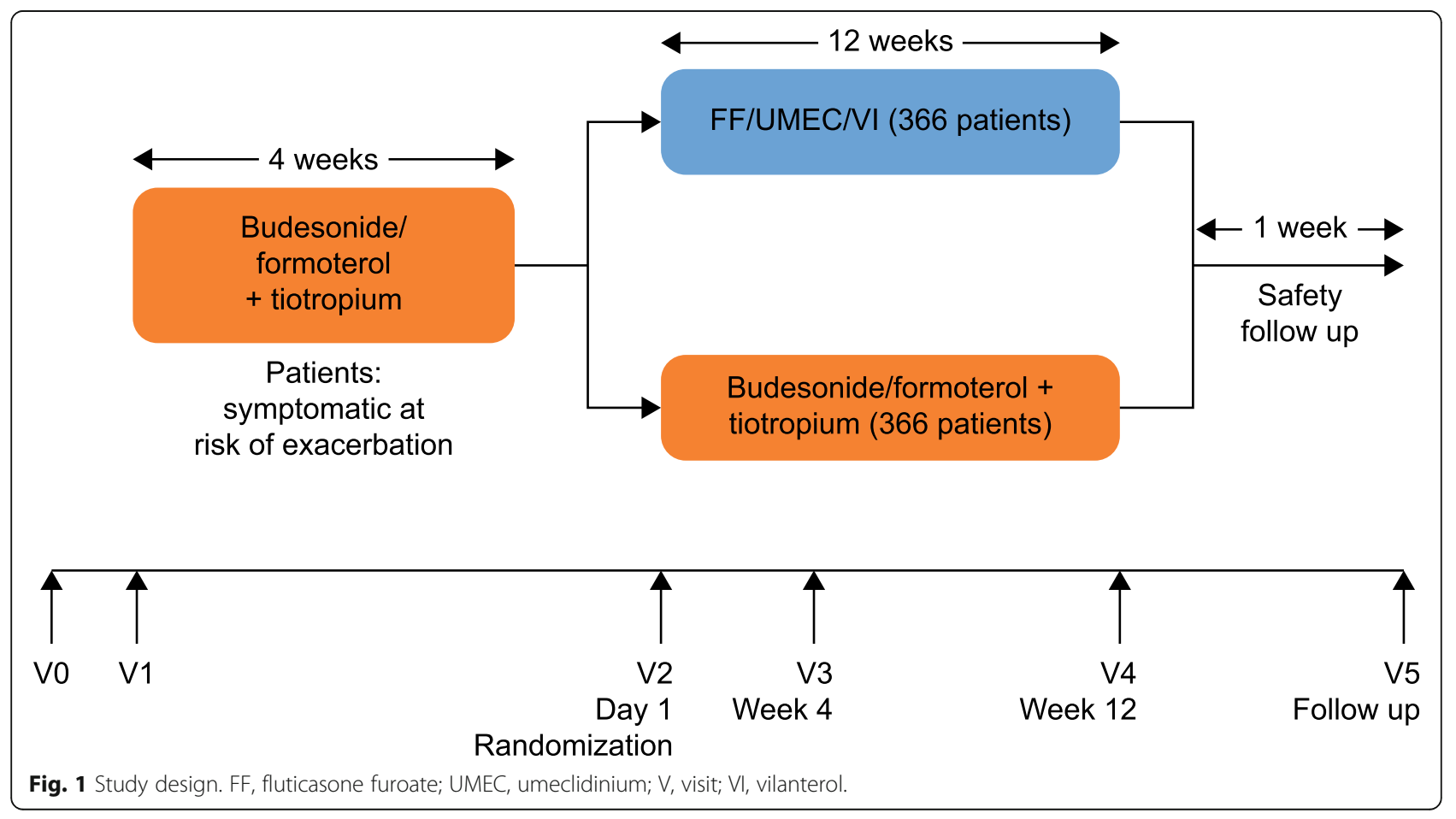


the electronic case report form (eCRF). Furthermore, during the 4-week run-in period patients were required to demonstrate $80-120 \%$ compliance with the run-in study medication, not experience any moderate or severe COPD exacerbations or pneumonia, and require no change in COPD medication. Full inclusion and exclusion criteria are provided in the Supplementary Materials.

\section{Study endpoints}

The primary endpoint was the weighted mean change from baseline in $\mathrm{FEV}_{1}$ over $0-24 \mathrm{~h}$ at Week 12 (Day 84). Spirometry was conducted at each study visit, with $\geq 3$ acceptable spirometry efforts obtained and the largest $\mathrm{FEV}_{1}$ measurement recorded. Secondary endpoints included the weighted mean change from baseline in $\mathrm{FEV}_{1}$ over $0-24 \mathrm{~h}$ on Day 1, and change from baseline in trough $\mathrm{FEV}_{1}$ on Days 2, 28, 84, and 85. Baseline was defined as the average of the two Day 1 pre-dose measurements. If one of the measurements was missing, the single remaining value was used as the baseline value. Other endpoints included the change from baseline in St George's Respiratory Questionnaire (SGRQ) total score and CAT score at Week 12, and the proportion of responders based on the SGRQ total score ( $\geq 4$-unit decrease from baseline) or CAT score ( $\geq 2$-unit decrease from baseline) at Week 12.

The incidence of adverse events (AEs), serious AEs (SAEs), and AEs of special interest (AESIs) were recorded at each study visit. AESIs are events associated with the known class effects for ICS, LABA, and LAMA treatment, which allows for a comprehensive review of safety outcomes that is not restricted by specific preferred terms.

A pre-specified pooled analysis of both studies was performed for the efficacy and safety endpoints, which also included subgroup analyses by age $(<65, \geq 65$ years), percent predicted $\mathrm{FEV}_{1}$ at screening $(\leq 30 \%, 30-<50 \%$, $\geq 50 \%)$, and baseline CAT score $(<20, \geq 20)$.

\section{Statistical analyses}

There were two analysis populations: the intent-to-treat (ITT) population included all randomized patients, except those randomized in error; the modified per protocol $(\mathrm{mPP})$ population included all patients in the ITT population except those with a protocol deviation of not meeting the inclusion, exclusion, or randomization criteria.

Estimands allow for the evaluation of study objectives while taking into account the occurrence of intercurrent events (events that preclude the observation of an endpoint or affect its interpretation). In these studies, the primary estimand based on the mPP population excluded data following the occurrence of these intercurrent events: discontinuation of randomized study treatment; taking the wrong randomized study treatment; taking a prohibited medication; unblinding of randomized study treatment; treatment non-compliance; moderate/severe COPD exacerbation or pneumonia. Estimand based on the ITT population excluded data only following the discontinuation of randomized study treatment. Estimands were used for the analysis of all efficacy endpoints.

The sample size calculations used a one-sided 2.5\% significance level and an estimate of residual standard deviation (SD) for 0-24-h weighted mean $\mathrm{FEV}_{1}$ of $230 \mathrm{~mL}$. A study with 620 evaluable patients would have $90 \%$ power to determine non-inferiority of FF/UMEC/VI to BUD/FOR+TIO based on 0-24-h weighted mean FEV at Week 12, with a margin of non-inferiority of $50 \mathrm{~mL}$, assuming a true mean treatment difference of $10 \mathrm{~mL}$. Approximately 732 patients were planned to be randomized assuming $10 \%$ premature discontinuation of study treatment and $5 \%$ protocol deviation.

Non-inferiority for the primary endpoint of $0-24-\mathrm{h}$ weighted mean $\mathrm{FEV}_{1}$ was analyzed in the $\mathrm{mPP}$ population using on-treatment data and a mixed model repeated measures (MMRM) analysis with covariates of baseline $\mathrm{FEV}_{1}$, visit, geographical region, treatment, visit by treatment, and visit by baseline interactions. If the lower bound of the two-sided 95\% confidence interval (CI) around the (FF/UMEC/VI vs BUD/FOR+TIO) treatment difference was above $-50 \mathrm{~mL}$ then $\mathrm{FF} / \mathrm{UMEC} / \mathrm{VI}$ would be considered non-inferior to $\mathrm{BUD} / \mathrm{FOR}+\mathrm{TIO}$. The primary endpoint was also analyzed in the ITT population. Only if non-inferiority was achieved with the primary analysis on the mPP population, would inference of superiority be made with the primary endpoint and other lung function endpoints on the ITT population. If superiority was not demonstrated for the primary endpoint, no inference would be drawn from $p$-values for treatment comparisons on secondary lung function endpoints in the ITT population. However, inference could still be drawn from $p$-values for treatment comparisons for other non-lung function endpoints (i.e. SGRQ and CAT) in the ITT population.

All other endpoints were assessed in the ITT population. The change from baseline in trough $\mathrm{FEV}_{1}$, serial $\mathrm{FEV}_{1}$, SGRQ total score, and CAT score was analyzed using a MMRM analysis with covariates of baseline value, geographical region, treatment, visit, visit by treatment, and visit by baseline interactions. The proportion of SGRQ total score and CAT score responders was analyzed using a generalized linear mixed model with a logit link function and covariates of treatment group, geographical region, visit, baseline, baseline by visit, and treatment by visit interactions. Safety endpoints were analyzed using descriptive statistics. The pre-specified pooled analysis of both studies was conducted using the pooled ITT population.

\section{Results}

\section{Study population}

The ITT population included 728 patients in Study 207608 (FF/UMEC/VI, $n=363$; BUD/FOR+TIO, $n=365$ ) 
(Fig. 2a), and 732 patients in Study 207609 (FF/UMEC/VI, $n=366$; BUD/FOR+TIO, $n=366$ ) (Fig. 2b). Of these, 720 patients in Study 207608 (FF/UMEC/VI, $n=358$; BUD/FOR+TIO, $n=362$ ) and 711 patients in Study 207609 (FF/UMEC/VI, $n=354$; BUD/FOR+TIO, $n=357$ ) were included in the mPP population. In total, 690 (95\%) and 700 (96\%) patients completed Study 207608 and Study 207609, respectively, with AEs or patient decision being the most common reasons for withdrawal (Fig. 2). Patient demographics and baseline characteristics were similar across treatment arms and studies (Table 1).

\section{Efficacy}

\section{Primary endpoint}

Once-daily FF/UMEC/VI demonstrated non-inferiority compared with twice-daily BUD/FOR plus once-daily TIO for the primary endpoint of weighted mean change from baseline in $0-24-\mathrm{hEV}_{1}$ at Week 12. Betweentreatment differences $(95 \% \mathrm{CI})$ in the $\mathrm{mPP}$ population were $15 \mathrm{~mL}(-13,43)$ in Study 207608 and $11 \mathrm{~mL}(-20$, 41) in Study 207609 (Table 2; Fig. 3). In the pooled analysis, the between-treatment difference was $14 \mathrm{~mL}(-5$, 34 ) in the ITT population (Table 2; Fig. 3).

\section{Secondary endpoint}

On Day 1, the weighted mean change from baseline in 0-24-h $\mathrm{FEV}_{1}$ was similar with $\mathrm{FF} / \mathrm{UMEC} / \mathrm{VI}$ and BUD/FOR+TIO in Study 207608 and 207609, and in the pooled analysis, in the ITT population (Table 3). On Day 84 and Day 85, change from baseline in trough $\mathrm{FEV}_{1}$ was numerically greater in patients receiving FF/UMEC/VI compared with BUD/FOR+TIO in Study 207608 and 207609, and in the pooled analysis, with the $95 \% \mathrm{CI}$ for treatment difference excluding zero (Table 3; Fig. 4). This improvement with FF/UMEC/VI versus BUD/FOR+TIO was seen from Day 28 in Study 207608 and from Day 2 in Study 207609 (Table 3).

\section{Other endpoints}

In the pooled analysis, an improvement in change from baseline in serial $\mathrm{FEV}_{1}$ at Day 1 was seen with FF/UMEC/VI compared with BUD/FOR+TIO at the 12-h time point, while BUD/FOR+TIO demonstrated improvement over FF/UMEC/VI at the 15-h time point (i.e. after administration of the second dose), as seen in both individual studies (Supplementary Figure 1). By Week 12 the change from baseline in serial $\mathrm{FEV}_{1}$ in the pooled analysis was in favor of FF/UMEC/VI at the 12-, 21-, 23-, and 24-h time points, with $95 \%$ CIs for treatment difference excluding zero; similar results were seen in each study (Fig. 5). BUD/FOR+TIO demonstrated an improvement over FF/UMEC/VI at the 15 -h time point (i.e. after administration of the second dose; Fig. 5).
In both studies and in the pooled analysis, no statistically significant between-treatment difference was seen in change from baseline in either SGRQ total score or CAT score or in the proportion of SGRQ total score responders or CAT score responders at Week 12 (Table 4, Supplementary Figures 2 and 3).

In the pooled analysis, a greater proportion of patients receiving $\mathrm{BUD} / \mathrm{FOR}+\mathrm{TIO}$ experienced at least one clinically important deterioration in trough $\mathrm{FEV}_{1}$ of $\geq 100 \mathrm{~mL}$ (BUD/FOR+TIO, 379/723 [52\%] patients; FF/UMEC/VI, 277/726 [38\%] patients).

\section{Subgroup analysis}

In the subgroup analyses of the pooled population by age, percent predicted $\mathrm{FEV}_{1}$ at screening or baseline CAT score, the results for weighted mean change from baseline in $0-24-\mathrm{hEV}_{1}$ at Week 12, and change in baseline in trough $\mathrm{FEV}_{1}$ at Day 84 and 85 were generally in line with the ITT population (Supplementary Figures 4 and 5). At Week 12, no treatment difference in the weighted mean change from baseline in 0-24-h $\mathrm{FEV}_{1}$ between FF/UMEC/VI and BUD/FOR+TIO was seen, with the exception of patients under 65 years of age (treatment difference: $31 \mathrm{~mL}$ ) and patients with baseline CAT score $\geq 20(29 \mathrm{~mL})$ in favor of FF/UMEC/VI (Supplementary Figure 4). When analyzing change from baseline in trough $\mathrm{FEV}_{1}$ at Day 84, FF/UMEC/VI demonstrated a treatment benefit compared with $\mathrm{BUD} / \mathrm{FOR}+\mathrm{TIO}$ in all subgroups except in patients with a $\mathrm{FEV}_{1} \leq 30 \%$ predicted at screening (Supplementary Figure 5A). A similar trend was seen at Day 85, although the treatment difference was generally less pronounced (Supplementary Figure 5B).

\section{Safety endpoints}

Overall, the incidence of AEs, SAEs, and AESIs were similar across treatments and both Study 207608 and 207609, including the incidence of cardiovascular effects and pneumonia AESI (Table 5). In Study 207608, 5 (1\%) and $6(2 \%)$ patients reported pneumonia AESI in the $\mathrm{FF} / \mathrm{UMEC} / \mathrm{VI}$ and BUD/FOR+TIO groups, respectively, while $2(<1 \%)$ patients with FF/UMEC/VI and $3(<1 \%)$ with BUD/FOR+TIO reported pneumonia AESI in Study 207609.

In Study 207608, a numerically higher number of patients in the FF/UMEC/VI group experienced lower respiratory tract infection (LRTI) excluding pneumonia AESI compared with patients in the BUD/FOR+TIO group (9 [2\%] vs $1[<1 \%]$, respectively). This was not seen in Study 207609, where $1(<1 \%)$ and $1(<1 \%)$ patients reported LRTI excluding pneumonia AESI in the FF/UMEC/VI and BUD/FOR+TIO groups, respectively. No LRTI excluding pneumonia serious AESIs were reported in either study for either treatment arm. 


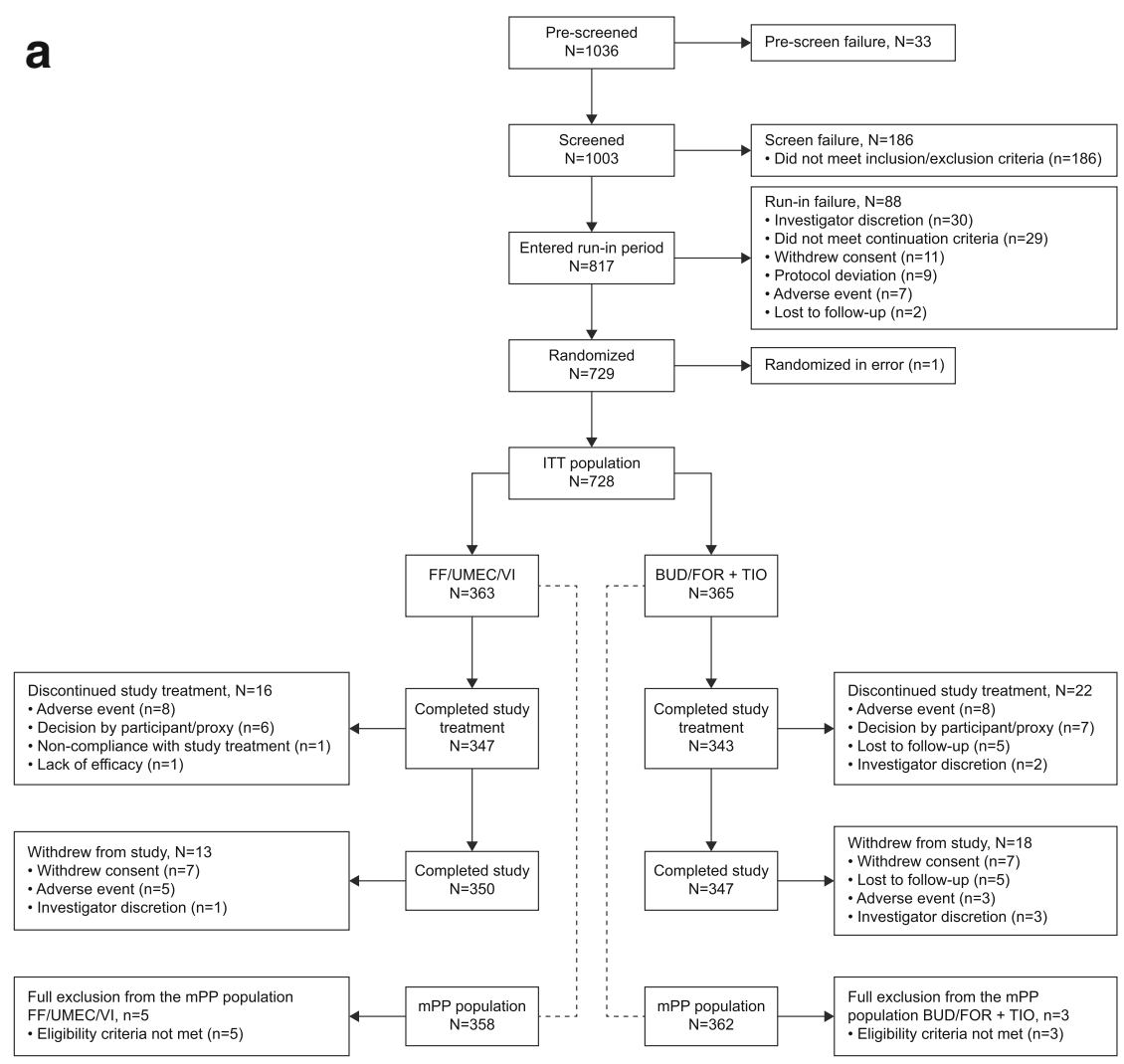

b

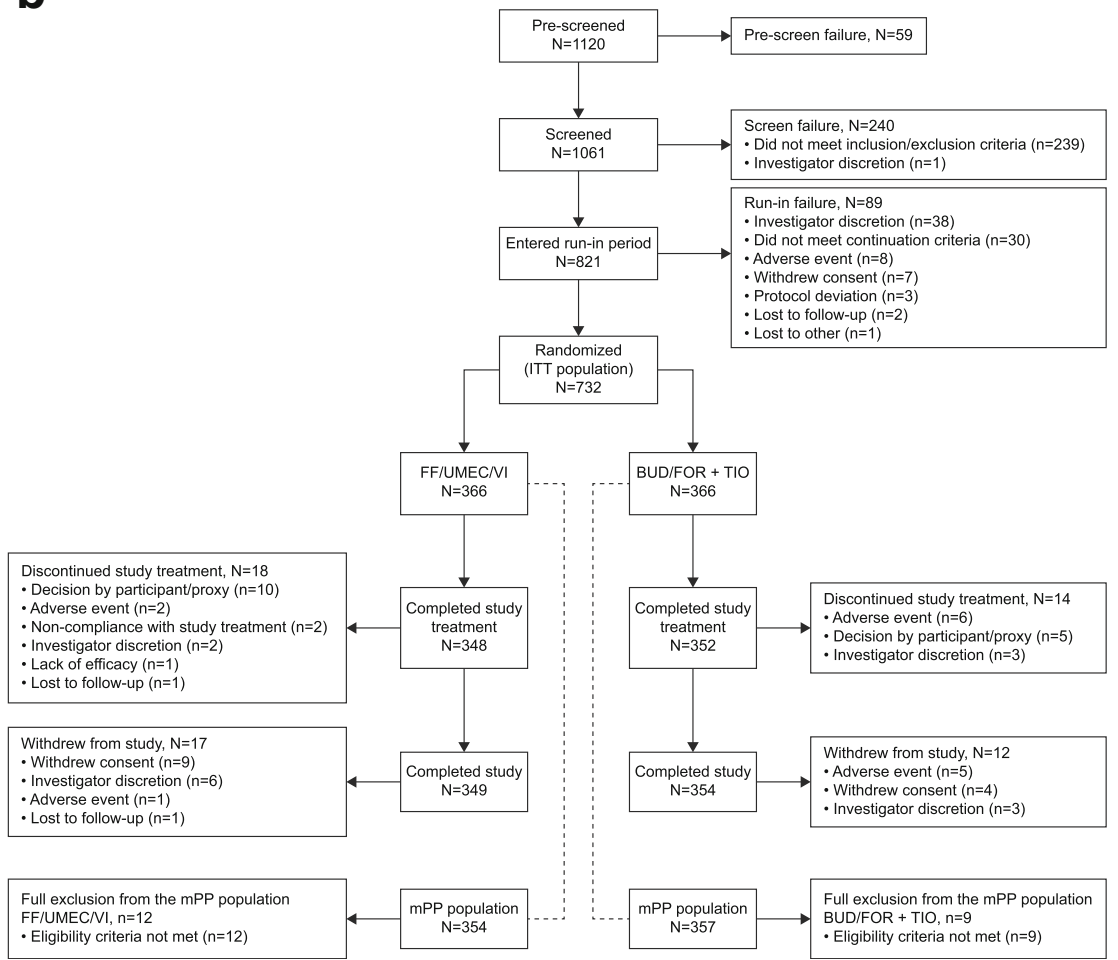

Fig. 2 Patient disposition for (a) Study 207608 and (b) Study 207609 (ITT population). BUD, budesonide; FF, fluticasone furoate; FOR, formoterol; ITT, intent-to-treat; mPP, modified per protocol; TIO, tiotropium; UMEC, umeclidinium; VI, vilanterol. 
Table 1 Patient demographics and baseline characteristics (ITT population)

\begin{tabular}{|c|c|c|c|c|}
\hline & Study 207608 & & Study 207609 & \\
\hline & $\begin{array}{l}\mathrm{FF} / \mathrm{MMEC} \mathrm{Nl} \\
N=363\end{array}$ & $\begin{array}{l}\text { BUD/FOR }+ \text { TIO } \\
N=365\end{array}$ & $\begin{array}{l}\text { FF/UMECNI } \\
N=366\end{array}$ & $\begin{array}{l}\text { BUD/FOR }+ \text { TIO } \\
N=366\end{array}$ \\
\hline Age, years, mean (SD) & $65.4(7.9)$ & $64.9(8.1)$ & $65.5(8.2)$ & $65.1(8.4)$ \\
\hline Female, n (\%) & $180(50)$ & $164(45)$ & $180(49)$ & $179(49)$ \\
\hline $\mathrm{BMI}, \mathrm{kg} / \mathrm{m}^{2}$, mean & $28.21(6.58)$ & $28.40(6.93)$ & $28.54(7.56)$ & $28.67(7.14)$ \\
\hline Smoking history and status & $n=363$ & $n=365$ & $n=366$ & $n=366$ \\
\hline Current smoker, n (\%) & $186(51)$ & $168(46)$ & $170(46)$ & $190(52)$ \\
\hline Smoking pack-years, mean (SD) & $47.9(25.3)$ & $47.9(25.7)$ & $48.3(23.8)$ & $50.2(24.6)$ \\
\hline COPD type & $n=356$ & $n=358$ & $n=362$ & $n=363$ \\
\hline Chronic bronchitis, n (\%) & $139(38)$ & $121(33)$ & $138(38)$ & $146(40)$ \\
\hline Emphysema, n (\%) & $102(28)$ & $133(36)$ & 118 (32) & $118(32)$ \\
\hline Chronic bronchitis and emphysema, $\mathrm{n}(\%)$ & $115(32)$ & $104(28)$ & $106(29)$ & $99(27)$ \\
\hline COPD history & $n=363$ & $n=365$ & $n=366$ & $n=365$ \\
\hline Duration of COPD, years, mean (SD) & $10.5(6.8)$ & $9.9(6.8)$ & $10.4(7.3)$ & $9.7(7.0)$ \\
\hline$<1$ year, $\mathrm{n}(\%)$ & $2(<1)$ & $4(1)$ & $5(1)$ & $4(1)$ \\
\hline$\geq 1-<5$ years, $n(\%)$ & $69(19)$ & $72(20)$ & $66(18)$ & $75(20)$ \\
\hline$\geq 5-<10$ years, $n(\%)$ & $123(34)$ & $133(36)$ & $126(34)$ & $152(42)$ \\
\hline$\geq 10$ years, $\mathrm{n}(\%)$ & $169(47)$ & $156(43)$ & $169(46)$ & $134(37)$ \\
\hline $\begin{array}{l}\text { Moderate COPD exacerbations in the previous } \\
12 \text { months, } n(\%)\end{array}$ & $n=363$ & $n=365$ & $n=366$ & $n=366$ \\
\hline 0 & $192(53)$ & $191(52)$ & $185(51)$ & $202(55)$ \\
\hline 1 & $60(17)$ & $55(15)$ & $67(18)$ & $59(16)$ \\
\hline$\geq 2$ & $111(31)$ & $119(33)$ & $114(31)$ & $105(29)$ \\
\hline $\begin{array}{l}\text { Severe COPD exacerbations in the previous } \\
12 \text { months, } n(\%)\end{array}$ & $n=363$ & $n=365$ & $n=366$ & $n=366$ \\
\hline 0 & $324(89)$ & $325(89)$ & $326(89)$ & $310(85)$ \\
\hline 1 & $34(9)$ & $35(10)$ & $37(10)$ & $47(13)$ \\
\hline$\geq 2$ & $5(1)$ & $5(1)$ & $3(<1)$ & $9(2)$ \\
\hline Screening lung function, mean (SD) & $n=363$ & $n=364$ & $n=362$ & $n=366$ \\
\hline Post-bronchodilator FEV 1 (L) & $1.176(0.431)$ & $1.199(0.411)$ & $1.128(0.398)$ & $1.181(0.429)$ \\
\hline Post-bronchodilator FEV $/$ /FVC (\% FEV 1 ) & $0.469(0.110)$ & $0.472(0.115)$ & $0.481(0.107)$ & $0.492(0.109)$ \\
\hline Post-bronchodilator $\mathrm{FEV}_{1}, \%$ predicted & $42.5(11.9)$ & $42.3(12.3)$ & $41.4(12.5)$ & $42.8(13.0)$ \\
\hline Peak inspiratory flow rate, $\mathrm{L} / \mathrm{min}^{*}$ & 208.37 (83.35) & $200.68(81.21)$ & $191.04(68.54)$ & $195.91(79.55)$ \\
\hline GOLD stage $\left(\%\right.$ predicted $\left.\mathrm{FEV}_{1}\right), \mathrm{n}(\%)$ & $n=363$ & $n=364$ & $n=362$ & $n=366$ \\
\hline Stage I ( $\geq 80 \%)$ & 0 & 0 & 0 & 0 \\
\hline Stage II ( $\geq 50$ to < 80\%) & $76(21)$ & $82(23)$ & $71(20)$ & $84(23)$ \\
\hline Stage III ( $\geq 30$ to < 50\%) & $236(65)$ & $226(62)$ & $219(60)$ & $221(60)$ \\
\hline Stage IV (<30\%) & $51(14)$ & $56(15)$ & $72(20)$ & $61(17)$ \\
\hline Reversibility to salbutamol ${ }^{\dagger}, \mathrm{n}(\%)$ & $n=358$ & $n=360$ & $n=358$ & $n=362$ \\
\hline Reversible & $65(18)$ & $80(22)$ & $48(13)$ & $55(15)$ \\
\hline CAT score at screening, mean (SD) ${ }^{\ddagger}$ & $21.6(6.5)$ & $22.0(6.6)$ & $22.2(6.3)$ & $22.3(6.4)$ \\
\hline COPD medications at screening, $\mathrm{n}(\%)$ & $n=363$ & $n=365$ & $n=366$ & $n=366$ \\
\hline$I C S+L A B A$ & $121(33)$ & $137(38)$ & $123(34)$ & $115(31)$ \\
\hline$I C S+L A M A+L A B A$ & $113(31)$ & $96(26)$ & $118(32)$ & $116(32)$ \\
\hline$\angle A B A+\angle A M A$ & $55(15)$ & $42(12)$ & $59(16)$ & $67(18)$ \\
\hline LAMA & $23(6)$ & 30 (8) & $26(7)$ & 31 (8) \\
\hline
\end{tabular}

*207608, FF/UMECNI: $n=348 ;$ BUD/FOR+TIO: $n=354 ; 207609$, FF/UMECNI: $n=342 ;$ BUD/FOR+TIO: $n=342 .{ }^{+}$Reversible defined as an increase in FEV ${ }_{1}$ of $\geq 12 \%$ and $\geq 200 \mathrm{~mL}$ following administration of salbutamol. ${ }^{\ddagger} 207608$, FF/UMECNI: $n=359$; BUD/FOR+TIO: $n=364 ; 207609$, FF/UMECNI: $n=364 ;$ BUD/FOR+TIO: $n=360$. BMI body mass index; BUD budesonide; CAT COPD Assessment Test; COPD chronic obstructive pulmonary disorder; $F E V$, forced expiratory volume in $1 \mathrm{~s}$; FF fluticasone furoate; FOR formoterol; FVC forced vital capacity; GOLD Global Initiative for Chronic Obstructive Lung Disease; ICS inhaled corticosteroid; ITT intent-to-treat; LABA long-acting $\beta_{2}$-agonist; LAMA long-acting muscarinic antagonist; SD standard deviation; TIO tiotropium; UMEC umeclidinium; VI vilanterol 
Table 2 Weighted mean FEV $10-24 \mathrm{~h}$ at Week 12

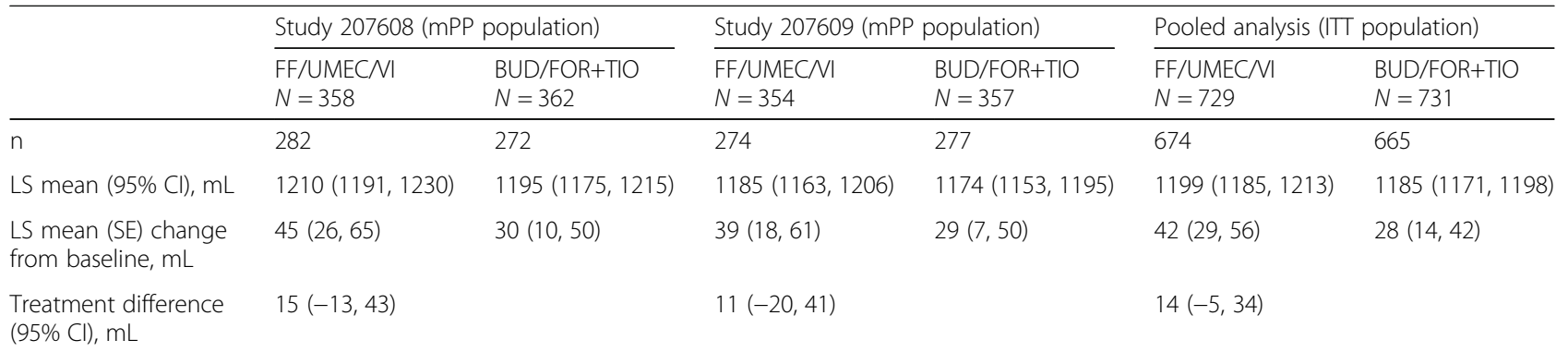

$n$ number of patients with analyzable data at the current time point

$B U D$ budesonide; $C I$ confidence interval; $F E V$ forced expiratory volume in $1 \mathrm{~s} ; F F$ fluticasone furoate; FOR formoterol; ITT intent-to-treat; $L S$ least squares; $m P P$ modified per protocol; SE standard error; TIO tiotropium; UMEC umeclidinium; VI vilanterol

No deaths were reported for patients receiving FF/UMEC/VI in either study, while 1 death was reported in the BUD/FOR+TIO group (lung adenocarcinoma) in Study 207609, not deemed to be related to study treatment.

\section{Discussion}

Studies 207608 and 207609 both compared singleinhaler FF/UMEC/VI therapy against the most commonly prescribed multiple-inhaler triple therapy in the USA, BUD/FOR+TIO, and met the study-defined primary endpoint of non-inferiority for once-daily FF/UMEC/VI versus twice-daily BUD/FOR plus once-daily TIO for change from baseline in weighted mean $\mathrm{FEV}_{1}$ over $0-24 \mathrm{~h}$ at Week 12 . No statistically significant differences between the treatments were seen in the change from baseline in SGRQ total score or CAT score at Week 12.

Of note, FF/UMEC/VI demonstrated improvements in the change from baseline in trough $\mathrm{FEV}_{1}$ at both Day 84 and 85 compared with BUD/FOR+TIO. Furthermore, results from the pooled serial $\mathrm{FEV}_{1}$ analysis indicate that FF/UMEC/VI was associated with greater improvements in lung function compared with BUD/FOR+TIO at specific time points within the $24 \mathrm{~h}$ (i.e. at both 12 and $24 \mathrm{~h}$ following the morning dose). These results suggest that, despite the perceived improvement after the second dose with twice-daily therapies [29], once-daily triple therapy with FF/UMEC/VI may reduce the variability of the treatment effect and thereby prevent the intermittent decline in lung function seen with twice-daily therapies $[30,31]$. The greater improvements in $\mathrm{FEV}_{1}$ with

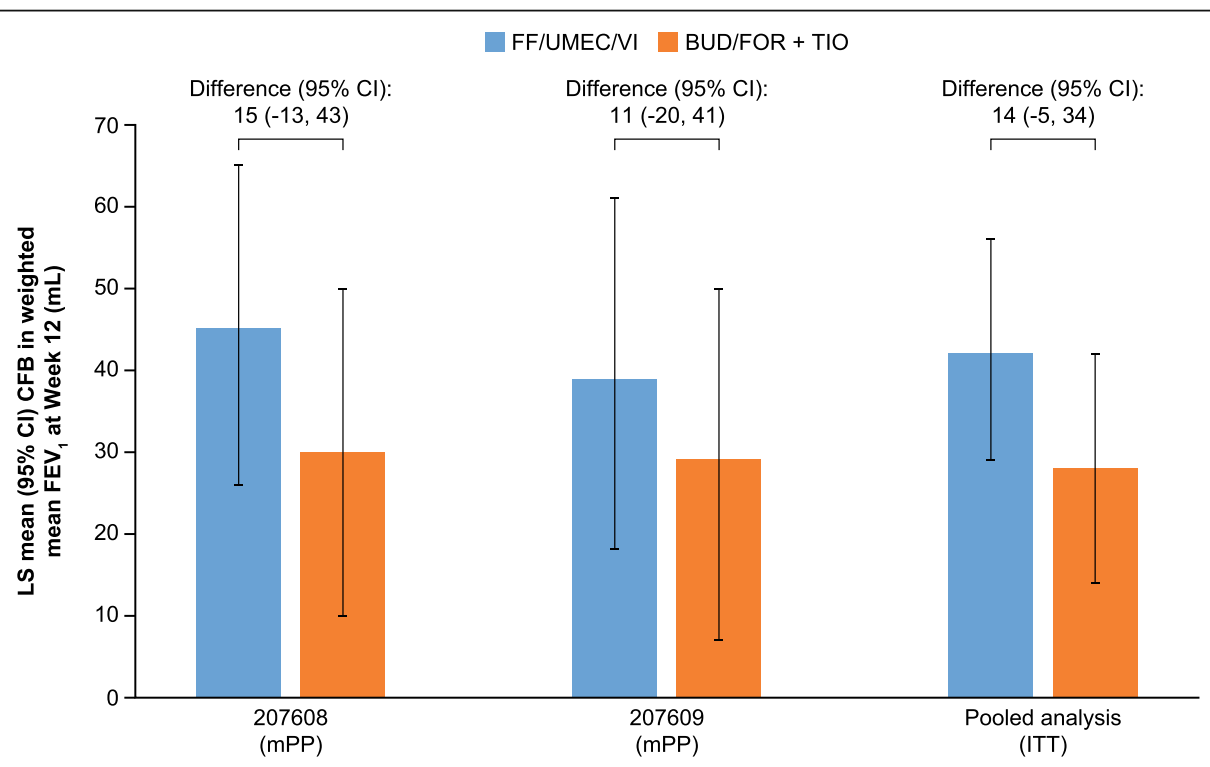

Fig. 3 Change from baseline in weighted mean FEV 1 over $0-24 \mathrm{~h}$ at Week 12. BUD, budesonide; CFB, change from baseline; $\mathrm{Cl}$, confidence interval; $F_{E V}$, forced expiratory volume in $1 \mathrm{~s}$; FF, fluticasone furoate; FOR, formoterol; ITT, intent-to-treat; LS, least squares; mPP, modified per protocol; TIO, tiotropium; UMEC, umeclidinium; VI, vilanterol. 


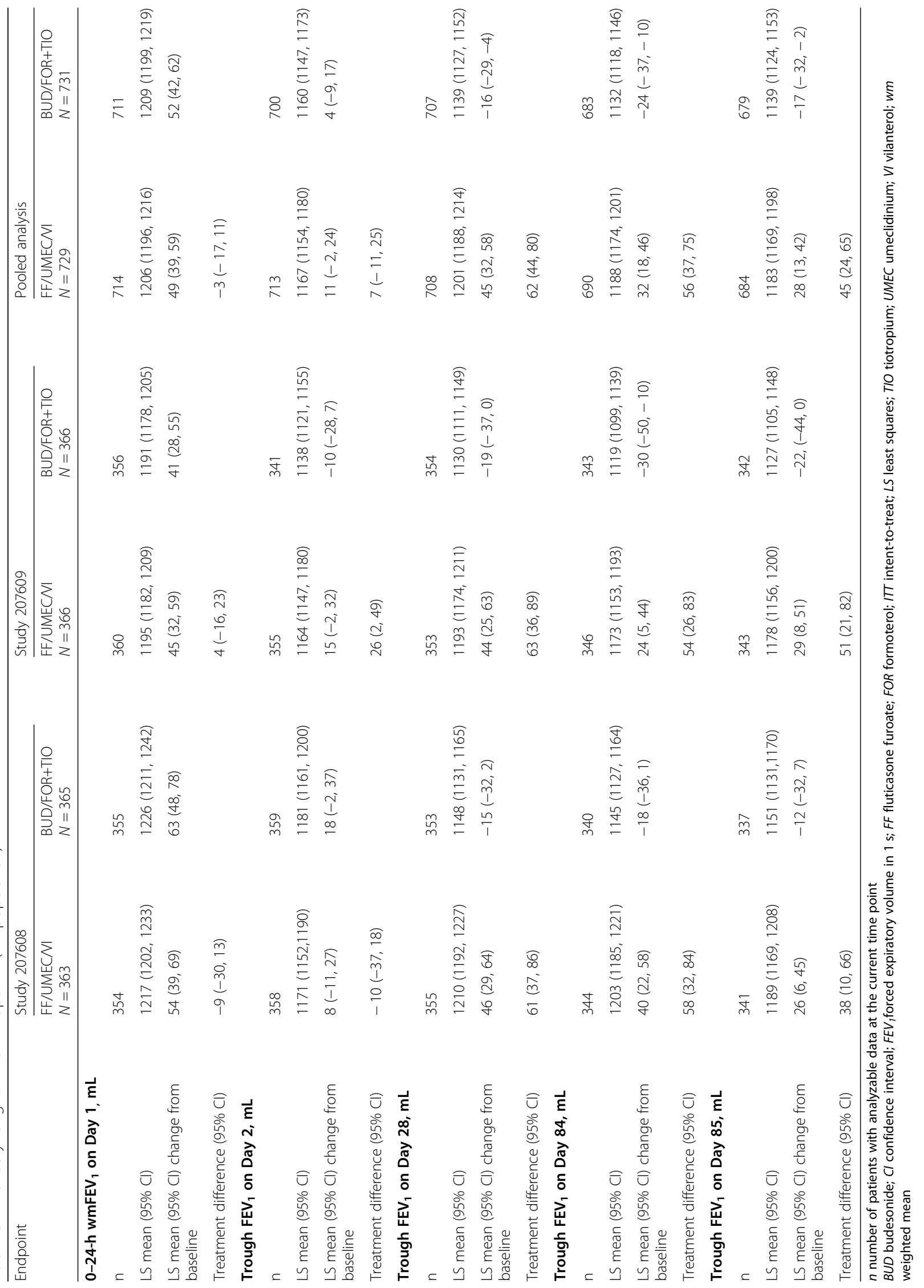




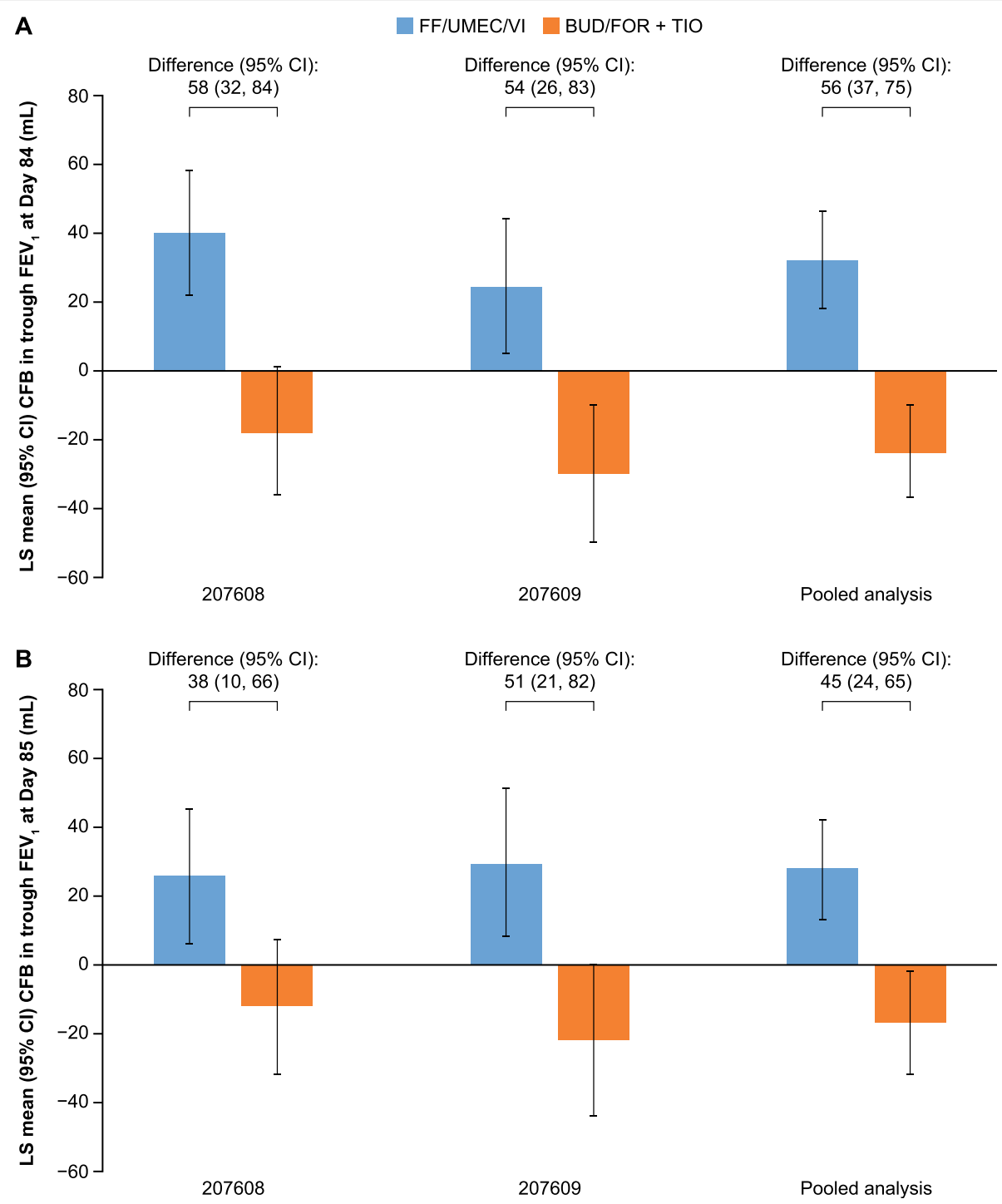

Fig. 4 Change from baseline in trough $\mathrm{FEV}_{1}$ at (a) Day 84; (b) Day 85 (ITT population). BUD, budesonide; CFB, change from baseline; Cl, confidence interval; $\mathrm{FEV}_{1}$, forced expiratory volume in $1 \mathrm{~s}$; FF, fluticasone furoate; FOR, formoterol; ITT, intent-to-treat; LS, least squares; TIO, tiotropium; UMEC, umeclidinium; VI, vilanterol.

FF/UMEC/VI at $12 \mathrm{~h}$ (i.e. prior to the second dose of $\mathrm{BUD} / \mathrm{FOR}+\mathrm{TIO})$ and at 21,23 , and $24 \mathrm{~h}$, suggest that patients who received BUD/FOR+TIO may be experiencing a loss of treatment effect followed by recovery to their baseline level following their additional required dose. This suggestion that FF/UMEC/VI may provide better protection against lung function deterioration is further supported by the lower proportion of patients experiencing a clinically important deterioration in trough $\mathrm{FEV}_{1}$ of $\geq 100 \mathrm{~mL}$ with $\mathrm{FF} / \mathrm{UMEC} / \mathrm{VI}$ compared with BUD/FOR+TIO.

Nighttime disturbances in patients with COPD are frequently reported, with over $65 \%$ of patients experiencing sleep disturbance owing to their symptoms [32, 33]. These nighttime disturbances are associated with patients having worse health status and worsening of COPD symptoms compared with patients who do not experience sleep disturbance [33]. Furthermore, it has also been reported that patients more frequently experience COPD symptoms that are worse than normal during the morning than any other time of day [34]. It is often thought that the administration of a second dose at night can alleviate these morning symptoms of COPD; however, the lack of difference in health status scores in this analysis suggest that there may be no difference between twice- and once-daily dosing for morning and nighttime symptom control. Nevertheless, the improvements seen in trough and serial $\mathrm{FEV}_{1}$ measures indicate that once-daily FF/UMEC/VI 


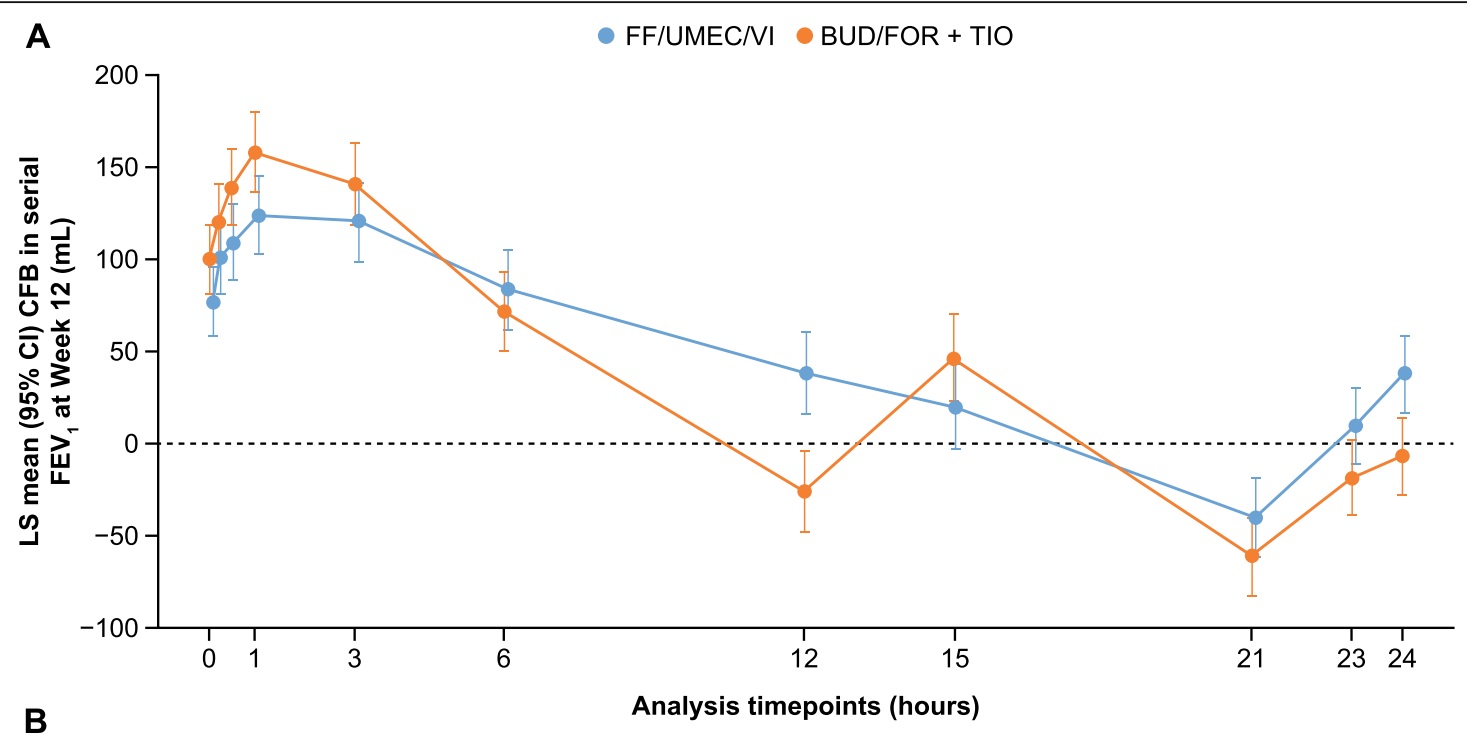

B

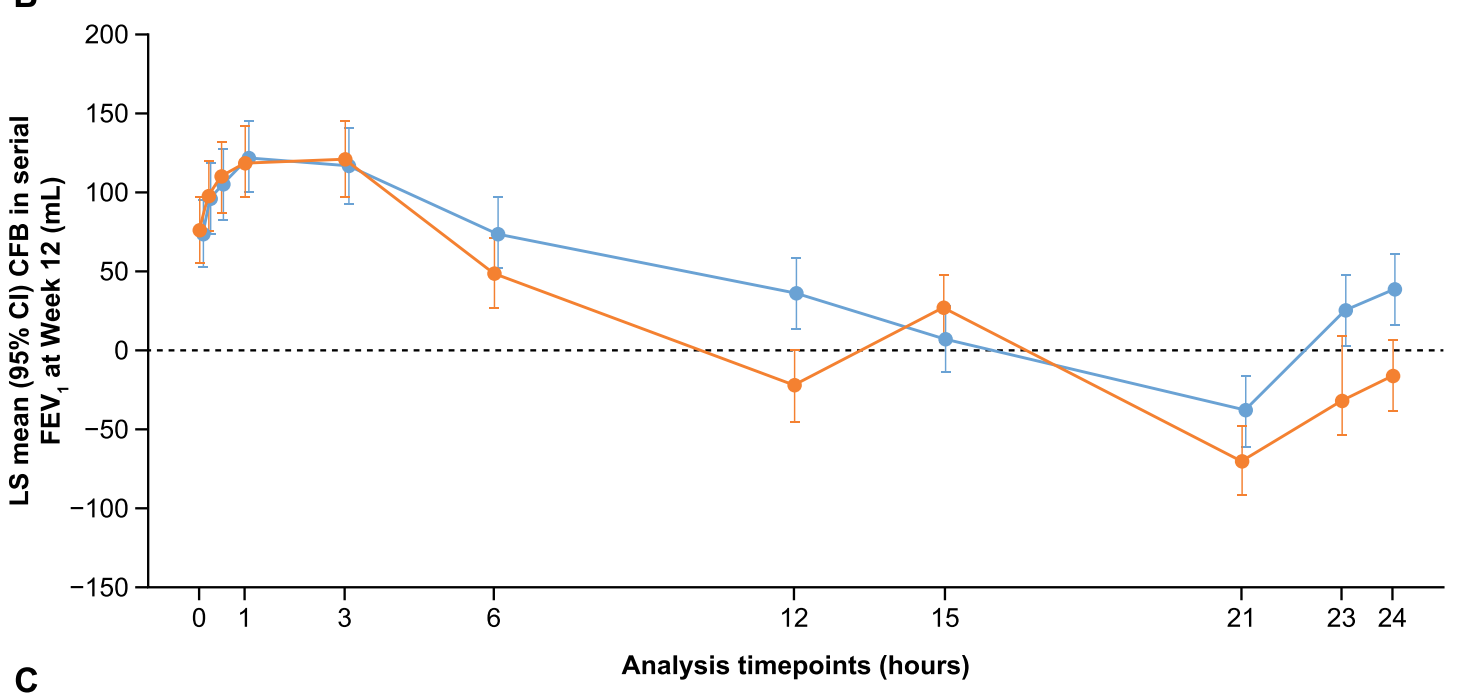

C

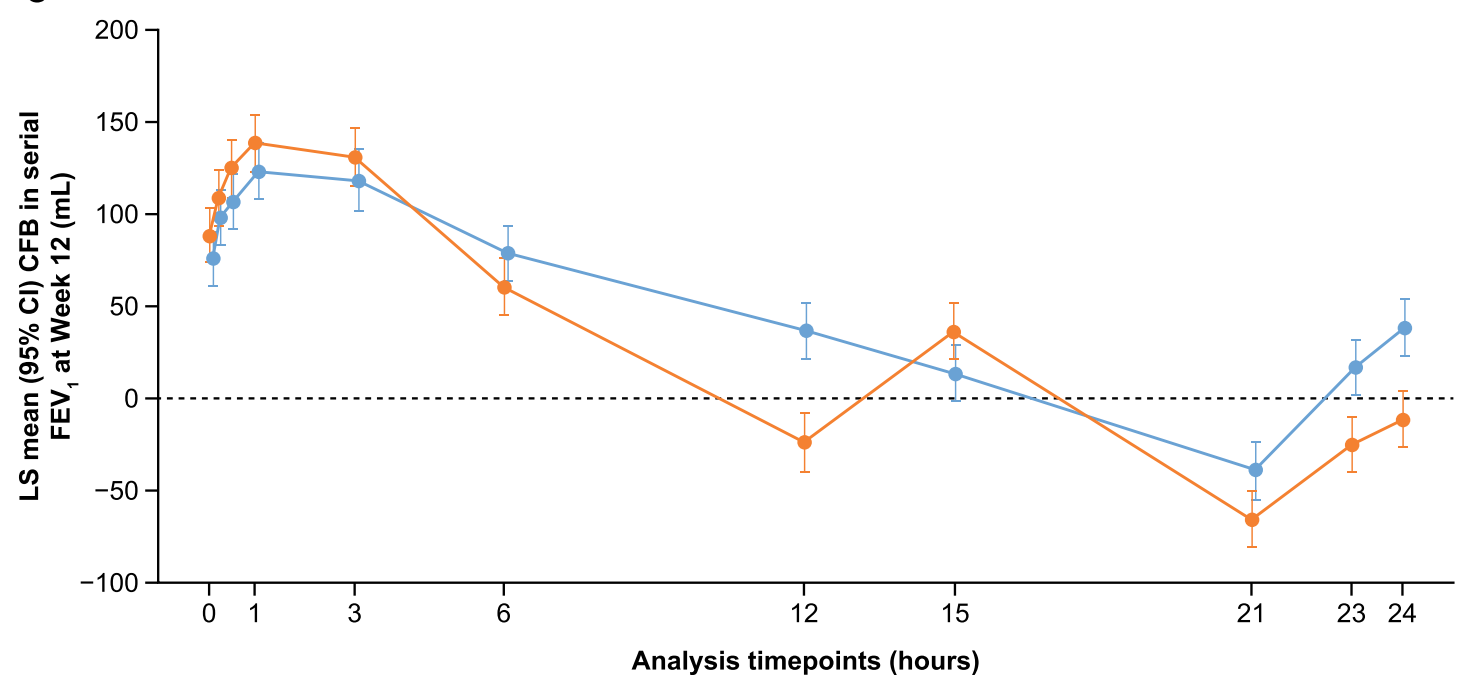

Fig. 5 Change from baseline in serial FEV 1 at Week 12 (ITT population). a. Study 207608; b. Study 207609; c. Pooled analysis. BUD, budesonide; Cl, confidence interval; $\mathrm{FEV}_{1}$, forced expiratory volume in $1 \mathrm{~s}$; FF, fluticasone furoate; FOR, formoterol; ITT, intent-to-treat; LS, least squares; TIO, tiotropium; UMEC, umeclidinium; VI, vilanterol. 


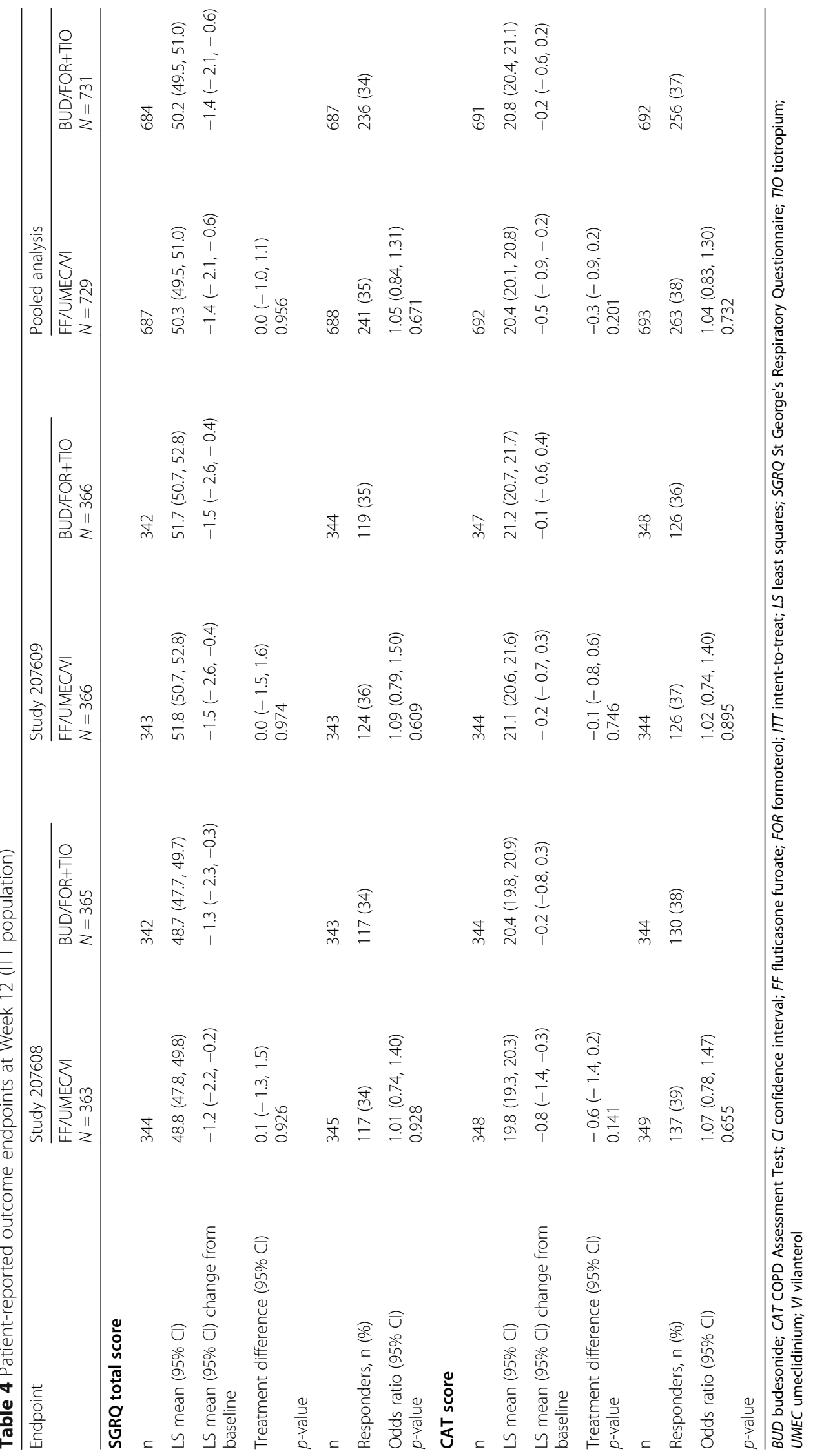


Table 5 Overall safety outcomes (ITT population)

\begin{tabular}{|c|c|c|c|c|c|c|c|c|}
\hline & \multicolumn{4}{|c|}{ Study 207608} & \multicolumn{4}{|c|}{ Study 207609} \\
\hline & \multicolumn{2}{|c|}{$\begin{array}{l}\text { FF/UMECNI } \\
N=363\end{array}$} & \multicolumn{2}{|c|}{$\begin{array}{l}\text { BUD/FOR+TIO } \\
N=365\end{array}$} & \multicolumn{2}{|c|}{$\begin{array}{l}\text { FF/UMECNI } \\
N=366\end{array}$} & \multicolumn{2}{|c|}{$\begin{array}{l}\text { BUD/FOR+TIO } \\
N=366\end{array}$} \\
\hline & n (\%) & Rate [\#] & n (\%) & Rate [\#] & n (\%) & Rate [\#] & n (\%) & Rate [\#] \\
\hline \multicolumn{9}{|l|}{ AEs } \\
\hline Any & $131(36)$ & $2932.8[244]$ & $121(33)$ & $2651.8[216]$ & $92(25)$ & $2488.8[205]$ & $109(30)$ & $2321.2[192]$ \\
\hline Drug-related & $23(6)$ & $372.6[31]$ & $16(4)$ & $233.3[19]$ & $9(2)$ & $182.1[15]$ & $10(3)$ & $145.1[12]$ \\
\hline $\begin{array}{l}\text { Leading to permanent } \\
\text { discontinuation or withdrawal }\end{array}$ & $7(2)$ & $132.2[11]$ & $7(2)$ & $85.9[7]$ & $2(<1)$ & $24.3[2]$ & $5(1)$ & $60.4[5]$ \\
\hline \multicolumn{9}{|l|}{ SAEs } \\
\hline Any & $25(7)$ & $444.7[37]$ & $14(4)$ & $221.0[18]$ & $12(3)$ & $279.2[23]$ & $17(5)$ & $266.0[22]$ \\
\hline Drug-related & $4(1)$ & $60.1[5]$ & 0 & & $1(<1)$ & $24.3[2]$ & $1(<1)$ & $12.1[1]$ \\
\hline $\begin{array}{l}\text { Leading to permanent } \\
\text { discontinuation or withdrawal }\end{array}$ & $5(1)$ & $96.2[8]$ & $5(1)$ & $61.4[5]$ & $1(<1)$ & $12.1[1]$ & $4(1)$ & $48.4[4]$ \\
\hline Fatal & 0 & & 0 & & 0 & & $1(<1)$ & $12.1[1]$ \\
\hline \multicolumn{9}{|l|}{ AESIs } \\
\hline Cardiovascular effects & $10(3)$ & $132.2[11]$ & $8(2)$ & $135.0[11]$ & $11(3)$ & 157.8 [13] & $8(2)$ & $120.9[10]$ \\
\hline $\begin{array}{l}\text { Decreased BMD and } \\
\text { associated fractures }\end{array}$ & $5(1)$ & $72.1[6]$ & $3(<1)$ & $36.8[3]$ & $2(<1)$ & $36.4[3]$ & $4(1)$ & $48.4[4]$ \\
\hline LRTI excluding pneumonia & $9(2)$ & $108.2[9]$ & $1(<1)$ & $12.3[1]$ & $1(<1)$ & $12.1[1]$ & $1(<1)$ & $12.1[1]$ \\
\hline Pneumonia & $5(1)$ & $60.1[5]$ & $6(2)$ & $73.7[6]$ & $2(<1)$ & $24.3[2]$ & $3(<1)$ & $60.4[5]$ \\
\hline
\end{tabular}

Rate is the number of events per 1000 patient-year, calculated as the number of events $\times 1000$ divided by the total treatment exposure $A E$ adverse event; $A E S I$ adverse event of special interest; $B M D$ bone mineral density; BUD budesonide; FF fluticasone furoate; FOR formoterol; ITT intent-to-treat; $L R T I$ lower respiratory tract infection; SAE serious adverse event; TIO tiotropium; UMEC umeclidinium; VI vilanterol

therapy may offer more consistent and sustained lung function benefits throughout the dosing interval than twice-daily BUD/FOR plus once-daily TIO.

Both treatment combinations were well tolerated, with low rates of treatment-related AEs or SAEs and few patients discontinuing owing to treatment-related AEs. A greater proportion of patients receiving FF/UMEC/VI experienced LRTI excluding pneumonia events compared with patients receiving BUD/FOR+TIO in Study 207608 and this higher incidence was driven primarily by bronchitis events $(7[2 \%]$ vs $1[<1 \%]$ of patients in FF/UMEC/VI and BUD/FOR+TIO arms, respectively). Of note, a greater proportion of patients with chronic bronchitis were enrolled to the FF/UMEC/VI arm compared with the BUD/FOR+TIO arm in Study 207608, whereas an equal proportion of patients with chronic bronchitis were enrolled to both treatment arms in Study 207609, where no difference in the incidence of LRTI excluding pneumonia events was observed. It is important to note that the incidence of cardiovascular or pneumonia AESIs, which have both been associated with these medication classes [35-38], were low and similar with both treatments and in both studies.

One limitation of these studies was the short period of assessment (12 weeks), which restricts any analysis of long-term safety outcomes and treatment effects (e.g. incidence of exacerbation events between the treatment combinations).

It is interesting to note that a greater treatment difference in trough $\mathrm{FEV}_{1}$ between FF/UMEC/VI and BUD/FOR+TIO was seen on Day 84 compared with Day 85. As treatment on Day 84 was self-administered at home, while on Day 85 it was administered in the clinic, this may suggest that some patients do not self-administer their second daily treatment dose at the optimal time, potentially reducing the effect of the treatment. Adherence is defined as not just conforming to the recommendations regarding the dosage and frequency of medication administration but also the timing of the administration [39]. Simplifying treatment regimens with a once-daily therapy has been suggested to improve adherence to therapy with respect to the timing of the medication compared with twice-daily therapy, which in turn may lead to improved clinical outcomes [19-21]. However, dummy inhalers were used in these studies to ensure blinding and, as such, the studies were not designed to assess the impact of single or multiple inhalers nor oncedaily or twice-daily therapy on adherence. Furthermore, the implementation of randomization criteria, to ensure that patients were $\geq 80$ to $\leq 120 \%$ compliant with the run-in study medication, maximized the adherence of the patient population and may therefore not be reflective of adherence in the general COPD population. It can 
reasonably be expected that the real-world treatment effect of these medications would reflect any impact of the dosing regimens on adherence, and future realworld or open-label studies to determine the impact of patient's adherence on treatment effectiveness may, therefore, reveal greater differences between treatments.

\section{Conclusion}

Once-daily single-inhaler triple therapy with FF/UMEC/VI provides similar overall improvements in weighted mean $\mathrm{FEV}_{1}$ and health status, and a similar safety profile, including a low incidence of pneumonia, as twice-daily multipleinhaler triple therapy with BUD/FOR+TIO. These results suggest that FF/UMEC/VI is a viable treatment option for patients who wish to simplify their treatment regimen from multiple- to single-inhaler triple therapy. The greater improvements with FF/UMEC/VI over BUD/FOR+TIO in trough $\mathrm{FEV}_{1}$ at 12 and $24 \mathrm{~h}$ further suggest that once-daily FF/UMEC/VI therapy may offer more consistent and sustained lung function benefits throughout the dosing interval compared with twice-daily BUD/FOR + once-daily TIO therapy.

\section{Supplementary information}

Supplementary information accompanies this paper at https://doi.org/10. 1186/s12931-020-01360-w.

Additional file 1. Full inclusion and exclusion criteria and supplementary figures $1-5$.

\section{Abbreviations}

AE: Adverse event; AESI: Adverse event of special interest; ATS: American Thoracic Society; BMI: Body mass index; BUD: Budesonide; CAT: COPD Assessment Test; CFB: Change from baseline; Cl: Confidence interval; COPD: Chronic obstructive pulmonary disease; ERS: European Respiratory Society; $F_{1}$ : Forced expiratory volume in $1 \mathrm{~s}$; FF: Fluticasone furoate; FOR: Formoterol; FVC: Forced vital capacity; GOLD: Global Initiative for Chronic Obstructive Lung Disease; ICS: Inhaled corticosteroid; ITT: Intent-totreat; LABA: Long-acting $\beta_{2}$-agonist; LAMA: Long-acting muscarinic antagonist; LRTI: Lower respiratory tract infection; MDI: Metered-dose inhaler; MMRM: Mixed measures repeated model; mPP: Modified per protocol; SAE: Serious adverse event; SD: Standard deviation; SGRQ: St George's Respiratory Questionnaire; TIO: Tiotropium; UMEC: Umeclidinium; VI: Vilanterol; wmCFB: Weighted mean change from baseline

\section{Acknowledgements}

Editorial support (in the form of writing assistance, assembling figures, collating author comments, grammatical editing, and referencing) was provided by Philip Chapman, at Fishawack Indicia Ltd., UK, and was funded by GSK.

\section{Authors' contributions}

The authors meet criteria for authorship as recommended by the International Committee of Medical Journal Editors, take responsibility for the integrity of the work as a whole, contributed to the writing and reviewing of the manuscript, and have given final approval for the version to be published. All authors had full access to the data in this study and take complete responsibility for the integrity of the data and accuracy of the data analysis. D Bernstein, GT Ferguson, and C Fogarty were involved in acquisition of data. N Brown, C Compton, MC Kaisermann, DA Lipson, and CQ Zhu were involved in the conception/design of the study and analysis/ interpretation of data. K Dorais was involved in the conception/design of the study. TC Corbridge, C Harvey, N Martin, M Stiegler, and F Sciurba were involved in the analysis/interpretation of data. The authors read and approved the final manuscript.

\section{Funding}

These studies were funded by GSK (study number 207608; NCT03478683; study number 207609; NCT03478696). The funders of the study had a role in the study design, data analysis, data interpretation, and writing of the report. ELLIPTA is owned by or licensed to the GSK Group of Companies.

Availability of data and materials

Anonymized individual participant data and study documents can be requested for further research from www.clinicalstudydatarequest.com.

\section{Ethics approval and consent to participate}

The study protocol, any amendments, the informed consent, and other information that required pre-approval were reviewed and approved by a national, regional, or investigational site ethics committee or institutional review board (IRB), in accordance with the International Council for Harmonisation of Technical Requirements for Registration of Pharmaceuticals for Human Use Good Clinical Practice and applicable country-specific requirements. All study patients provided written informed consent.

\section{Consent for publication}

Not applicable.

\section{Competing interests}

N Brown, C Compton, TC Corbridge, K Dorais, C Harvey, MC Kaisermann, DA Lipson, N Martin, M Stiegler, and C-Q Zhu are employees of GlaxoSmithKline (GSK) and are shareholders in GSK. GT Ferguson received grants, personal fees, and non-financial support from Boehringer Ingelheim, Novartis, AstraZeneca, Pearl Therapeutics, Sunovion, Theravance, and GSK, grants and personal fees from Verona, and Sanofi, and personal fees from Mylan, Innoviva, and Circassia, and grants from Altavant, unrelated to this work. C Fogarty has nothing to disclose. F Sciurba received research support from the COPD Foundation, Department of Defense Beta Blocker, Gala Therapeutics, Inc., GSK, NIH, Nuvaira, PCORI, PneumRX, Inc., PulmonX, and ResMed Corp, participated in advisory board for GSK, PneumRX, Inc., Theravance, and Verona and previously received research support from Astellas and AstraZeneca, unrelated to this work. D Bernstein has received grants and personal fees from GSK and ALK America, grants from Aimmune, Amgen, AstraZeneca, Genentech, Merck, Mylan, Novartis, Pearl, Shire, Teva, Adare, Knopp, Leo, Mandala, Gossamer, and Regeneron, and personal fees from Gerson Lehman, Guidepoint Global, and Covis, unrelated to this work.

\section{Author details}

'Pulmonary Research Institute of Southeast Michigan, Farmington Hills, MI, USA. ${ }^{2}$ GlaxoSmithKline, Stockley Park, Uxbridge, Middlesex, UK. ${ }^{3}$ GlaxoSmithKline, Brentford, Middlesex, UK. ${ }^{4}$ GlaxoSmithKline, Research Triangle Park, NC, USA. ${ }^{5}$ Northwestern University, Feinberg School of Medicine, Chicago, IL, USA. ${ }^{6}$ GlaxoSmithKline, Collegeville, PA, USA.

${ }^{7}$ Spartanburg Medical Research, Spartanburg, SC, USA. ${ }^{8}$ Perelman School of Medicine, University of Pennsylvania, Philadelphia, PA, USA. ${ }^{9}$ University of Leicester, Leicester, UK. ${ }^{10}$ University of Pittsburgh Medical Center, Pittsburgh, PA, USA. ${ }^{11}$ University of North Carolina at Chapel Hill, Chapel Hill, NC, USA.

${ }^{12}$ Bernstein Clinical Research Center and Division of Immunology, Allergy and Rheumatology, University of Cincinnati College of Medicine, Cincinnati, $\mathrm{OH}$, USA.

Received: 17 December 2019 Accepted: 12 April 2020

Published online: 29 May 2020

\section{References}

1. Global Initiative for Chronic Obstructive Lung Disease. Global Initiative for Chronic Obstructive Lung Disease 2020. Available at https://goldcopd.org/. Last Accessed March 2020

2. IQVIA. Applied Patient Level Data, weekly March 2018-March 2019. 2019.

3. Aaron SD, Vandemheen $\mathrm{KL}$, Fergusson $\mathrm{D}$, et al. Tiotropium in combination with placebo, salmeterol, or fluticasone-salmeterol for treatment of chronic 
obstructive pulmonary disease: a randomized trial. Ann Intern Med. 2007; 146:545-55.

4. Frith PA, Thompson PJ, Ratnavadivel R, et al. Glycopyrronium once-daily significantly improves lung function and health status when combined with salmeterol/fluticasone in patients with COPD: the GLISTEN study, a randomised controlled trial. Thorax. 2015;70:519-27.

5. Hanania NA, Crater GD, Morris AN, et al. Benefits of adding fluticasone propionate/salmeterol to tiotropium in moderate to severe COPD. Respir Med. 2012;106:91-101.

6. Jung KS, Park HY, Park SY, et al. Comparison of tiotropium plus fluticasone propionate/salmeterol with tiotropium in COPD: a randomized controlled study. Respir Med. 2012;106:382-9.

7. Siler TM, Kerwin E, Tombs L, Fahy WA, Naya I. Triple therapy of Umeclidinium + inhaled corticosteroids/long-acting Beta2 agonists for patients with COPD: pooled results of randomized placebo-controlled trials. Pulm Ther. 2016;2:43-58.

8. Siler TM, Kerwin E, Singletary K, Brooks J, Church A. Efficacy and safety of Umeclidinium added to fluticasone propionate/Salmeterol in patients with COPD: results of two randomized. Double-Blind Studies COPD. 2016;13:1-10.

9. Singh D, Brooks J, Hagan G, Cahn A, O'Connor BJ. Superiority of "triple" therapy with salmeterol/fluticasone propionate and tiotropium bromide versus individual components in moderate to severe COPD. Thorax. 2008;63:592-8.

10. Welte T, Miravitlles M, Hernandez $P$, et al. Efficacy and tolerability of budesonide/formoterol added to tiotropium in patients with chronic obstructive pulmonary disease. Am J Respir Crit Care Med. 2009:180:741-50.

11. Short PM, Williamson PA, Elder DHJ, et al. The impact of tiotropium on mortality and exacerbations when added to inhaled corticosteroids and long-acting beta-agonist therapy in COPD. Chest. 2012;141:81-6.

12. Vanfleteren L, Fabbri LM, Papi A, Petruzzelli S, Celli B. Triple therapy (ICS/ LABA/LAMA) in COPD: time for a reappraisal. Int J Chron Obstruct Pulmon Dis. 2018;13:3971-81.

13. Humenberger M, Horner A, Labek A, et al. Adherence to inhaled therapy and its impact on chronic obstructive pulmonary disease (COPD). BMC Pulm Med. 2018;18:163.

14. Rand CS. Patient adherence with COPD therapy. Eur Respir Rev. 2005;14:97-101.

15. Restrepo RD, Alvarez MT, Wittnebel LD, et al. Medication adherence issues in patients treated for COPD. Int J Chron Obstruct Pulmon Dis. 2008;3:371-84.

16. George M. Adherence in asthma and COPD: new strategies for an old problem. Respir Care. 2018;63:818-31.

17. DiMatteo MR. Variations in patients' adherence to medical recommendations: a quantitative review of 50 years of research. Med Care. 2004:42:200-9.

18. van Boven JF, Chavannes NH, van der Molen T, et al. Clinical and economic impact of non-adherence in COPD: a systematic review. Respir Med. 2014; 108:103-13.

19. Bogart M, Stanford RH, Laliberte F, et al. Medication adherence and persistence in chronic obstructive pulmonary disease patients receiving triple therapy in a USA commercially insured population. Int J Chron Obstruct Pulmon Dis. 2019;14:343-52.

20. Yu AP, Guerin A, Ponce de Leon D, et al. Therapy persistence and adherence in patients with chronic obstructive pulmonary disease: multiple versus single long-acting maintenance inhalers. J Med Econ. 2011;14:486-96.

21. Gaduzo S, McGovern V, Roberts J, Scullion JE, Singh D. When to use singleinhaler triple therapy in COPD: a practical approach for primary care health care professionals. Int J Chron Obstruct Pulmon Dis. 2019;14:391-401.

22. Ferguson GT, Rabe KF, Martinez FJ, et al. Triple therapy with budesonide/ glycopyrrolate/formoterol fumarate with co-suspension delivery technology versus dual therapies in chronic obstructive pulmonary disease (KRONOS): a double-blind, parallel-group, multicentre, phase 3 randomised controlled trial. Lancet Respir Med. 2018;6:747-58.

23. Lipson DA, Barnacle $H$, Birk $R$, et al. FULFIL trial: once-daily triple therapy for patients with chronic obstructive pulmonary disease. Am J Respir Crit Care Med. 2017;196:438-46

24. Lipson DA, Barnhart F, Brealey N, et al. Once-daily single-inhaler triple versus dual therapy in patients with COPD. N Engl J Med. 2018;378:1671-80.

25. Papi A, Vestbo J, Fabbri L, et al. Extrafine inhaled triple therapy versus dual bronchodilator therapy in chronic obstructive pulmonary disease (TRIBUTE): a double-blind, parallel group, randomised controlled trial. Lancet. 2018;391: 1076-84.

26. Singh D, Papi A, Corradi $M$, et al. Single inhaler triple therapy versus inhaled corticosteroid plus long-acting beta2-agonist therapy for chronic obstructive pulmonary disease (TRILOGY): a double-blind, parallel group, randomised controlled trial. Lancet. 2016;388:963-73.

27. Vestbo J, Papi A, Corradi M, et al. Single inhaler extrafine triple therapy versus long-acting muscarinic antagonist therapy for chronic obstructive pulmonary disease (TRINITY): a double-blind, parallel group, randomised controlled trial. Lancet. 2017:389:1919-29.

28. Celli BR, MacNee W, Force AET. Standards for the diagnosis and treatment of patients with COPD: a summary of the ATS/ERS position paper. Eur Respir J. 2004:23:932-46.

29. Di Marco F, Santus P, Scichilone N, et al. Symptom variability and control in COPD: advantages of dual bronchodilation therapy. Respir Med. 2017;125:49-56

30. Sethi S, Kerwin E, Watz H, et al. AMPLIFY: a randomized, phase III study evaluating the efficacy and safety of aclidinium/formoterol vs monocomponents and tiotropium in patients with moderate-to-very severe symptomatic COPD. Int J Chron Obstruct Pulmon Dis. 2019;14:667-82.

31. Beeh KM, Derom E, Echave-Sustaeta J, et al. The lung function profile of once-daily tiotropium and olodaterol via Respimat((R)) is superior to that of twice-daily salmeterol and fluticasone propionate via Accuhaler((R)) (ENERGITO((R)) study). Int J Chron Obstruct Pulmon Dis. 2016;1 1:193-205.

32. Agusti A, Hedner J, Marin JM, et al. Night-time symptoms: a forgotten dimension of COPD. Eur Respir Rev. 2011:20:183-94.

33. Ding B, Small M, Bergstrom G, Holmgren U. A cross-sectional survey of night-time symptoms and impact of sleep disturbance on symptoms and health status in patients with COPD. Int J Chron Obstruct Pulmon Dis. 2017; 12:589-99.

34. Partridge MR, Karlsson N, Small IR. Patient insight into the impact of chronic obstructive pulmonary disease in the morning: an internet survey. Curr Med Res Opin. 2009;25:2043-8.

35. Rogliani P, Calzetta L, Matera MG, et al. Inhaled therapies and cardiovascular risk in patients with chronic obstructive pulmonary disease. Expert Opin Pharmacother. 2019;20:737-50

36. Salpeter SR, Ormiston TM, Salpeter EE. Cardiovascular effects of betaagonists in patients with asthma and COPD: a meta-analysis. Chest. 2004; 125:2309-21.

37. Crim C, Dransfield MT, Bourbeau J, et al. Pneumonia risk with inhaled fluticasone furoate and vilanterol compared with vilanterol alone in patients with COPD. Ann Am Thorac Soc. 2015;12:27-34

38. Kew KM, Dias S, Cates CJ. Long-acting inhaled therapy (beta-agonists, anticholinergics and steroids) for COPD: a network meta-analysis. Cochrane Database Syst Rev. 2014:CD010844. https://doi.org/10.1002/14651858. CD010844.pub2

39. Cramer JA, Roy A, Burrell A, et al. Medication compliance and persistence: terminology and definitions. Value Health. 2008;11:44-7.

\section{Publisher's Note}

Springer Nature remains neutral with regard to jurisdictional claims in published maps and institutional affiliations.

Ready to submit your research? Choose BMC and benefit from:

- fast, convenient online submission

- thorough peer review by experienced researchers in your field

- rapid publication on acceptance

- support for research data, including large and complex data types

- gold Open Access which fosters wider collaboration and increased citations

- maximum visibility for your research: over $100 \mathrm{M}$ website views per year

At BMC, research is always in progress.

Learn more biomedcentral.com/submission 